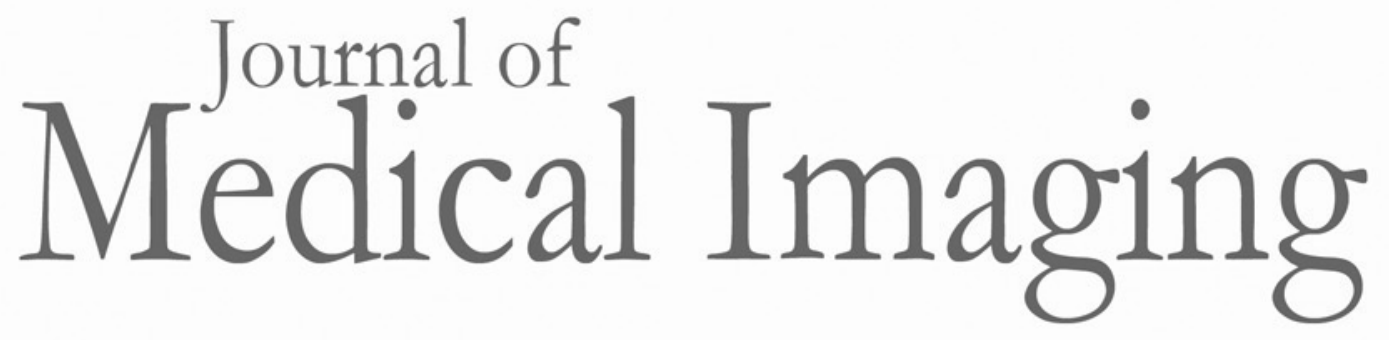

Medicallmaging.SPIEDigitalLibrary.org

\title{
Multicell migration tracking within angiogenic networks by deep learning-based segmentation and augmented Bayesian filtering
}

\author{
Mengmeng Wang \\ Lee-Ling Sharon Ong \\ Justin Dauwels \\ H. Harry Asada
}




\title{
Multicell migration tracking within angiogenic networks by deep learning-based segmentation and augmented Bayesian filtering
}

\author{
Mengmeng Wang, ${ }^{a, *}$ Lee-Ling Sharon Ong, ${ }^{b}$ Justin Dauwels, ${ }^{c}$ and H. Harry Asada ${ }^{d}$ \\ ${ }^{a}$ Nanyang Technological University, Energy Research Institute, Singapore \\ ${ }^{b}$ Singapore-MIT Alliance for Research and Technology, Singapore \\ 'Nanyang Technological University, School of Electrical and Electronic Engineering, Singapore \\ 'Massachusetts Institute of Technology, Department of Mechanical Engineering, Cambridge, Massachusetts, United States
}

\begin{abstract}
Cell migration is a key feature for living organisms. Image analysis tools are useful in studying cell migration in three-dimensional (3-D) in vitro environments. We consider angiogenic vessels formed in 3-D microfluidic devices (MFDs) and develop an image analysis system to extract cell behaviors from experimental phase-contrast microscopy image sequences. The proposed system initializes tracks with the end-point confocal nuclei coordinates. We apply convolutional neural networks to detect cell candidates and combine backward Kalman filtering with multiple hypothesis tracking to link the cell candidates at each time step. These hypotheses incorporate prior knowledge on vessel formation and cell proliferation rates. The association accuracy reaches $86.4 \%$ for the proposed algorithm, indicating that the proposed system is able to associate cells more accurately than existing approaches. Cell culture experiments in 3-D MFDs have shown considerable promise for improving biology research. The proposed system is expected to be a useful quantitative tool for potential microscopy problems of MFDs. ( The Authors. Published by SPIE under a Creative Commons Attribution 3.0 Unported License. Distribution or reproduction of this work in whole or in part requires full attribution of the original publication, including its DOI. [DOI: 10.1117/1.JMI.5.2.024005]
\end{abstract}

Keywords: backward Kalman filters; coarse time-lapse phase-contrast images; convolutional neural networks; endothelial cell networks tracking; end-point confocal images; multiple hypothesis tracking

Paper 18025R received Feb. 7, 2018; accepted for publication May 17, 2018; published online Jun. 13, 2018.

\section{Introduction}

Cell migration experiments can be performed in three-dimensional (3-D) microfluidic devices (MFDs). ${ }^{1}$ These devices, with channels allowing either fluid to flow or gel scaffolds to be injected, mimic the in vivo environments. In conventional two-dimensional (2-D) in vitro experiments, cells move freely and form a thin layer rather than a structure. In in vivo experiments, we can only see the formed structure. However, from the experiments in the 3-D MFDs, we can obtain the microscopy images of both the structure and the individual cells. As controlled reactions can be reproduced with a small volume of samples and reagents, MFDs are used for long-term studies of many biological processes. In this study, we consider cell migration during angiogenesis and develop an automated image analysis system to extract cell migration behaviors.

Angiogenesis is the formation of new blood vessels from preexisting vessels. ${ }^{2}$ It is a critical process in growth, development, and cancer invasion. During angiogenesis, endothelial cells (ECs) lining an existing vessel (monolayer) will sprout out into the gel, in response to local chemical and mechanical stimuli. ${ }^{3-5}$ Our angiogenic experiments are conducted in MFDs, where the ECs have been shown to migrate in 3-D environments by Ong et al. ${ }^{6}$ Stained cells under fluorescent/confocal microscopy provide clear cell locations. However, staining inhibits

*Address all correspondence to: Mengmeng Wang, E-mail: mengmeng@ntu .edu.sg cell proliferation and migration especially over long period. ${ }^{7}$ Therefore, we culture the unstained ECs and observe the angiogenic vessels by phase-contrast microscopy ${ }^{8}$ at $20 \times$ magnification daily over a period of 10 to 14 days. ${ }^{9}$ Each image has a resolution of $1532 \times 2048$ pixels. At the end point, we image the vessels by confocal microscopy after staining cell nuclei with Hoechst dye (Invitrogen) and actin with Alexa Fluor 488 Phalloidin (Invitrogen).

Automated image analysis tools to extract cell migration behaviors are useful for a wide range of biomedical research. Conventional image analysis systems focus on cell migration in 2-D in vitro environments, whereas our cells are cultured in MFDs. Unlike the cells in 2-D, our cells migrate within the 3-D collagen gel, remodel the gel, and form 3-D blood vessels. Moreover, these cells do not overtake each other due to the existence of tight cell junctions, and their migration is constrained by the vessels. This creates a unique problem that has never been addressed.

\subsection{Existing Approaches}

The existing automated image analysis techniques for cell segmentation and tracking can be classified into two categories: tracking by model evolution and tracking by detection. The former category detects and tracks the cells simultaneously by representing the cells with mathematical models, whereas the latter category first detects cell candidates in all the frames independently and then links the detected candidates into tracks. 


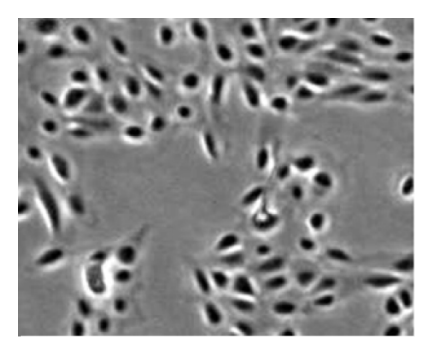

(a)

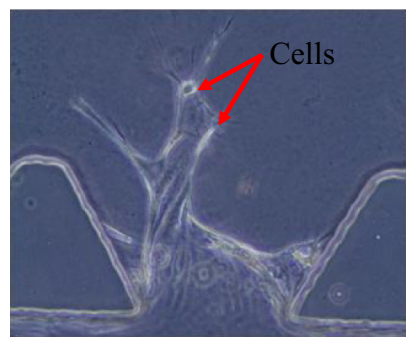

(b)
Fig. 1 Examples of cells (a) on a 2-D surface and (b) within 3-D angiogenic vessels (red arrows point to cells).

In model evolution algorithms, the mathematically represented cell contours are smoothly evolved from one time frame to the next one, using a velocity term defined by the content of the "target frame." rithms require a high imaging frequency to allow sufficient cell overlap between frames and accurate cell contour/boundary detection. These approaches are not applicable to our images due to our low imaging frequency (one day) and unclear cell boundaries (tight cell junctions).

In tracking by detection algorithms, cells are first detected in all the frames independently using thresholding of pixel intensities, ${ }^{15,16}$ edge detection, ${ }^{17}$ image restoration, ${ }^{18-20}$ or template matching. ${ }^{21}$ Kanade et al. ${ }^{18}$ applied image restoration to segment the cells on a 2-D flat surface from phase-contrast images, as shown in Fig. 1(a). Due to the different culture environments, the morphologies of our cells within angiogenic vessels are different [see Fig. 1(b)]. Moreover, the thickness of the MFDs is $120 \mu \mathrm{m}$, whereas the thickness of a cell on a flat surface is around 3 to $20 \mu \mathrm{m}$. Due to the artifacts of phase-contrast microscopy, imaging thicker specimens produces severe halo effects that reduce the visibility of our cell boundaries unlike the data by Kanade et al. ${ }^{18}$ In addition to that, some cells may be slightly out of focus. Therefore, the image restoration technique is not applicable for our images. A different approach is template matching; a template is compared to patches of a graylevel image by means of a match statistics evaluated for each patch. ${ }^{22}$ Euclidean distance, mean square difference, and support vector machine (SVM) can work as the template matching criteria for cell detection. ${ }^{21,23-26}$ Since we can easily obtain cell templates from end-point phase-contrast images by aligning the latter with their corresponding end-point confocal images, we apply convolutional neural networks $(\mathrm{CNN})$ to detect cell candidates from the time-lapse phase-contrast images.

Subsequently, association algorithms, such as "nearestneighborhood" association, ${ }^{27,28}$ mean-shift process, ${ }^{29-31}$ the Hungarian method, ${ }^{32}$ Viterbi algorithm, ${ }^{33}$ Kalman filtering, ${ }^{34}$ particle filtering, ${ }^{35}$ multiple hypothesis tracking (MHT), ${ }^{18,36,37}$ and active structured learning, ${ }^{38}$ can be used to determine the most likely cell correspondence between frames. These techniques are either applied to images of cells migrating on a 2-D surface or 3-D in vivo florescence images, which are far different from our 3-D in vitro application. In the works by Ong et al., ${ }^{6}$ stained ECs and vessels were tracked using Bayesian filtering from time-lapse confocal images of 30-min intervals. However, our images are acquired once a day, which is much longer than most existing tracking systems. Therefore, tracking these cells is a challenge that has not been previously addressed.

\subsection{Summary of Contribution}

In this paper, we focus on tracking by detection algorithm to track the migrating ECs within angiogenic vessels formed in 3-D MFDs by linking the coarse time-lapse 2-D phase-contrast images and 3-D end-point confocal images. Since the ECs in 3D MFDs can appear and disappear from our 2-D phase-contrast images, the main challenge is to accurately identify the cell candidates and track cell division and migration in 3-D (in focus/out of focus) over image sequences.

Our proposed system includes image registration, vessel segmentation, cell detection, and multiple hypothesis Kalman filtering, to track cell proliferation and migration within angiogenic vessels in different frames. As mentioned, we obtain time-lapse phase-contrast images and end-point confocal images of the angiogenic vessels from the experiments. The stained cells are easily recognized in the end-point confocal images. Therefore, instead of starting with the first time point like other cell tracking applications, we apply backward Kalman filter by initializing our tracks with the accurate cell locations obtained from the confocal images at the final time point where all proliferation has occurred. Furthermore, we incorporate biological knowledge to add constraints and to estimate the track probability during cell association:

- In our angiogenic vessels, cells cannot overtake the cells in front of them, whereas cells on a 2-D surface can move freely. This difference is one of the constraints during association.

- In conventional approaches, the increment of cells with time is only due to proliferation. However, for angiogenic experiments in 3-D MFDs, cells can migrate from cell-gel interface to form the angiogenic vessels. In other words, the increment of cells can be either due to proliferation or migration from cell-gel interface. Thus, we include an empirical equation in our tracking algorithm to estimate the probability that one cell is due to migration from cell-gel interface.

Since one phase-contrast image is recorded each day for each slot, biologists can conduct many experiments in parallel, where cell viabilities are well-maintained. Moreover, by backward tracking each cell in the 3-D network structure, cell lineage and trajectory information, linking the chemical and physical characteristics of a cell can be obtained in an automated manner.

The rest of this paper is structured as follows. In Sec. 2, we explain our proposed automated cell tracking system. In Sec. 3, we present numerical results on our experimental phase-contrast images. We offer concluding remarks in Sec. 4.

\section{Automated Multicell Tracking System}

Our automated tracking system is shown in Fig. 2. First, we align and transform all the experimental images, including both phase-contrast images and the end-point confocal images, into one common coordinate system through image registration. We then apply morphological image processing algorithms to segment the binary vessel shape. This shape is converted to a medial axis transform (MAT) representation. ${ }^{39}$ Simultaneously, we train a CNN classifier to detect and label cell candidates in the phase-contrast images. Last, by combining backward Kalman filtering with MHT, we associate and track the detected cell candidates over sequences. From the tracking 


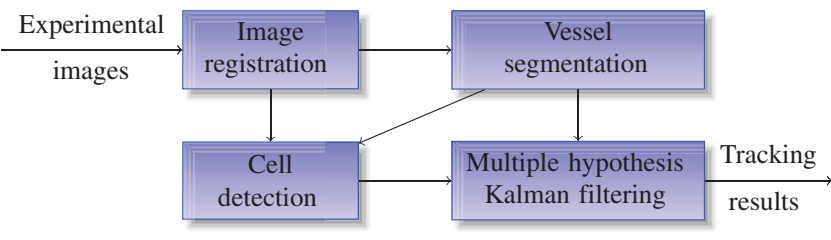

Fig. 2 Diagram of the proposed automated tracking system: image registration to align all the experimental images, vessel segmentation to represent binary vessel shape, cell detection to label all cell candidates, and multiple hypothesis Kalman filtering to associate the detected cell candidates.

results, we can obtain cell lineage and trajectory information to understand cell characteristics better. In the following, we will discuss each component in detail.

\subsection{Image Registration}

We acquired the experimental images manually at different days; hence, the angiogenic vessels are misaligned across the image sequences as shown in Fig. 3. We spatially register
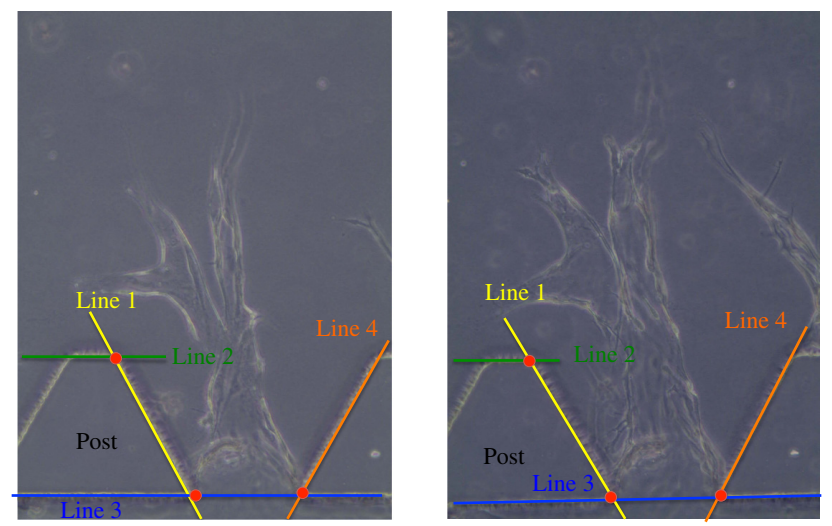

Fig. 3 Experimental phase-contrast images of two successive frames. The trapezoidal post is designed to retain the gel within the microfluidic channel. Lines 1 to 4 can be detected and represented using Hough transform. The red points are the correspondence points used during image registration. all the image sequences by transforming them into a common coordinate system, so that we can associate and track all the cells.

To register these images, we first extract salient features from all the images. We choose the intersections of the straight post edges (lines 1 to 4 ) as features, which are labeled in red in Fig. 3. To automatically detect these features, we apply the Hough transform to identify the straight lines representing the post edges. From these detected lines, the corresponding intersections between images are obtained. We then derive the scale, linear translation, and rotation parameters based on the intersections' coordinates by iterative closest point to align all the images. The pixels below the identified post edges are marked to zero to avoid any post features to be detected as vessels or cells in the later stage.

\subsection{Vessel Segmentation}

We aim to investigate the behavior of cells within the gel. Hence, we do not need to segment cells under the cell-gel interface [see Fig. 4(a)]. Based on the observations, we fit a parabola to estimate the cell-gel interface in the experimental images. The parabola equation has the following form:

$a_{1}\left(x-a_{p}+a_{3}\right)^{2}+a_{2}$,

where $a_{p}$ is the intersection point of lines 1 and 4 obtained during image registration, and $a_{1}, a_{2}$, and $a_{3}$ are the coefficients of the parabola. The best-fit is obtained by brute-force optimization. ${ }^{1}$ The pixels below the parabola are masked out as our region of interest is the area above the cell-gel interface.

Next, we convert the processed experimental images into binary images with the global image threshold computed using Otsu's ${ }^{40}$ method. We obtain the segmented binary vessel shape [see Fig. 4(b)] through the following series of morphological operations in MATLAB ${ }^{\mathrm{TM}}:^{22}$

- "imclose" to connect the gaps in the boundaries: structuring element is a 2-D disk-shaped structuring element with 15 pixels,

- "imfill" to fill the holes inside the vessel area, and

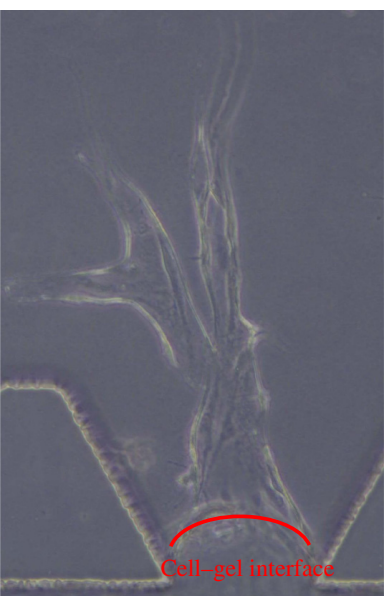

(a)

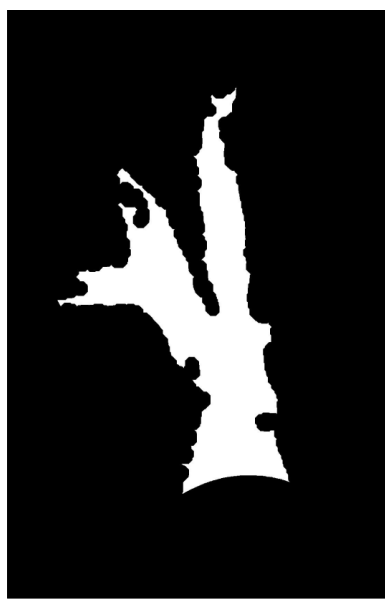

(b)

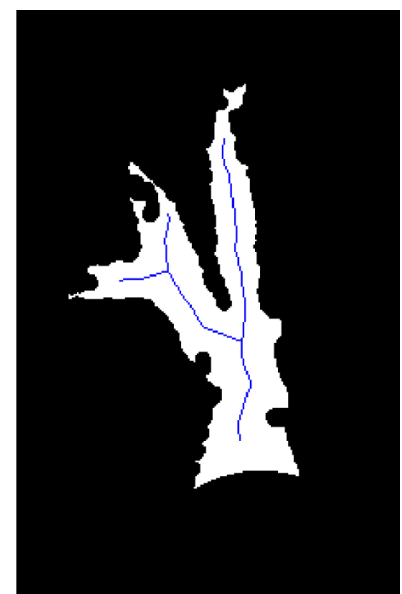

(c)

Fig. 4 Examples of vessel segmentation: (a) cell-gel interface, (b) the segmented binary vessel shape, and (c) vessel shape with centerline. 
- "bwareaopen" to remove the noises that have fewer than $P$ pixels: $P=20,000$.

These parameters are selected based on a set of training images, and moderate changes in these parameters do not lead to significant change in the binary vessel shapes.

We represent the segmented vessels shape with MAT by a centerline [see Fig. 4(c)] and radii. ${ }^{39}$ We can reconstruct the original vessel shape for visualization with the obtained MAT data set. Since the cells typically remain within the vessel, we can eliminate cell candidates outside the vessel during cell detection.

\subsection{Cell Detection}

$\mathrm{CNN}$ is a widely used deep learning approach in pattern-recognition and image-recognition problems. ${ }^{41}$ We construct a CNN architecture by stacking multiple layers (see Fig. 5) and train the CNN network to distinguish cell and noncell templates. The resulting CNN classifier is then used to detect the cell candidates from the time-lapse phase-contrast images.

We use the end-point confocal images, where we can easily recognize the cells, to select our cell templates to ensure they are informative. By aligning the phase-contrast images with their corresponding confocal images, we can distinguish cell coordinates and noncell coordinates in the phase-contrast images. These coordinates are cropped as our templates, as shown in Fig. 6. After rotating the templates by $90 \mathrm{deg}, 180 \mathrm{deg}$, and $270 \mathrm{deg}$, respectively, flipping them horizontally and vertically, and adding Gaussian noise, we obtain 3228 cell templates and 3372 noncell templates to train the CNN classifier. Each template has a size of $100 \times 100$, which is sufficiently large to fit each individual cell.

The architecture of a CNN varies depending on the types and numbers of layers included. Some common types of layers include convolution, ReLU, pooling, dropout, fully connected, softmax, and classification layer.
The convolution layers are the core building blocks of a CNN, which extract different features of the input. ${ }^{41,42}$ An image becomes a stack of filtered images after a convolution layer. ReLU is the abbreviation of rectified linear unit, which implements the function $y=\max (x, 0)$ to set any negative input value to zero. It increases the nonlinear properties of the overall network without affecting the receptive fields of the convolution layer. ${ }^{43}$ The pooling layer serves to progressively reduce the spatial size of the representation, to reduce the number of parameters and amount of computation in the network and, hence, also to control overfitting. Max-pooling is the most common pooling operation, which partitions the input image into a set of nonoverlapping rectangles, and for each such subregion, outputs the maximum. After several convolution, ReLU, and max-pooling layers, the fully connected layer combines all of the features learned by the previous layers to classify the images. ${ }^{44} \mathrm{~A}$ dropout layer, which randomly sets input elements to zero with a given probability, follows the fully connected layer to reduce overfitting. ${ }^{45}$ The fully connected layer usually uses the softmax activation function (softmax layer) for a classification problem. ${ }^{46}$ The classification layer is the final layer, which uses the probabilities returned by the softmax activation function for each input to assign it to one of the classes.

The types and number of layers included in a CNN classifier depend on the training data. To get the most suitable architecture of our CNN classifier, we vary not only the number of layers but also the parameters of each layer. For the data at hand, we apply fivefold cross validation to get the optimal CNN architecture. The training templates are randomly partitioned into five equal-sized subsamples, where one subsample is retained as the validation data for testing the classifier and the remaining four subsamples are used as training data. We repeat the crossvalidation process for five times to obtain our CNN classifier, which yields a classification accuracy of $92.39 \%$, as shown in Fig. 5. The first convolutional layer has 20 filters with a filter

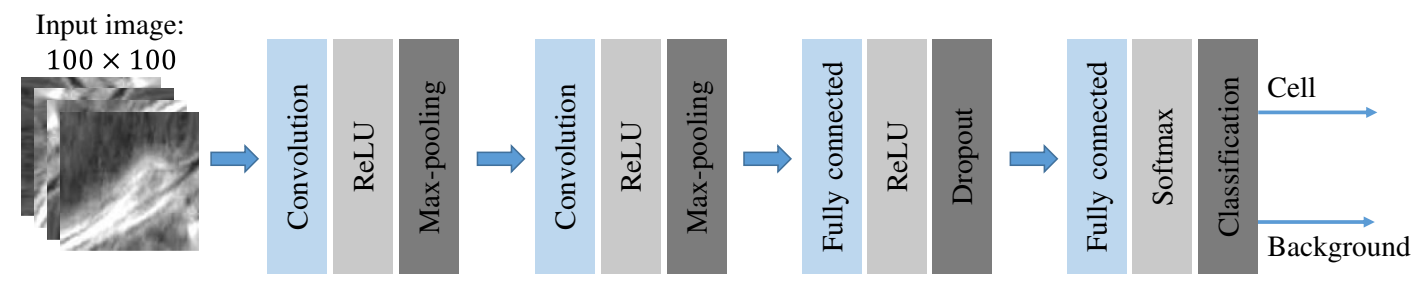

Fig. 5 Architecture of the CNN classifier for cell detection.
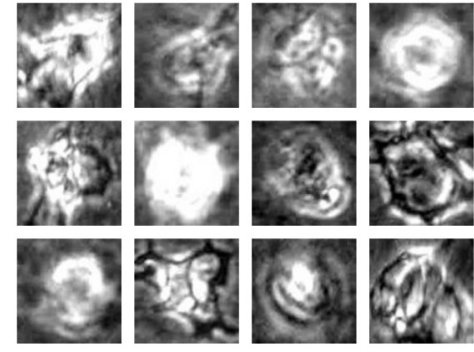

(a)
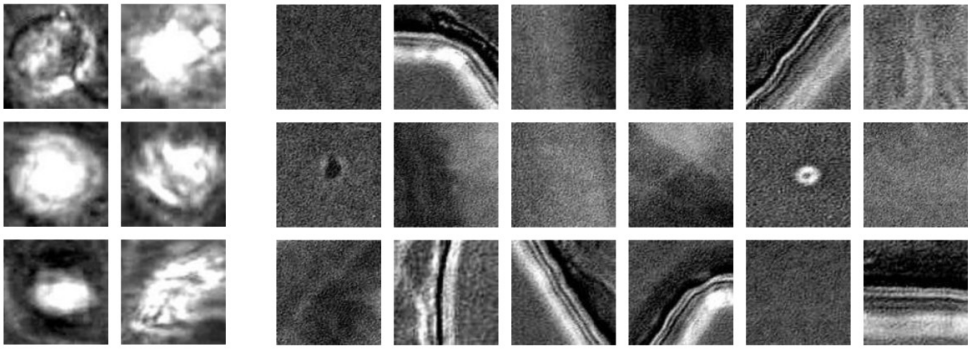

(b)

Fig. 6 Examples of (a) cell and (b) noncell templates from phase-contrast images to train the CNN classifier. Size of each template is $100 \times 100$. 
size of $5 \times 5$, and the second convolutional layer has 20 filters with a filter size of $10 \times 10$. The pool size of both max-pooling layers is $2 \times 2$. The output sizes of the first and second fully connected layers are 10 and 2 , respectively.

We then apply the resulting CNN classifier to classify the pixels in our experimental phase-contrast images through a sliding window manner. The centers of the clusters, which are classified as positive class, are selected as the position of cell nuclei.

\subsection{Multiple Hypothesis Kalman Filtering}

We combine backward Kalman filtering with MHT to associate and track the cell candidates within the vessels over time. Instead of starting with the image at the first time point, we initialize our tracks with the end-point confocal image, which provide the accurate cell locations and where all proliferation has occurred. Furthermore, we combine biological knowledge, such as vessel information and cell proliferation rate, to add constraints when estimating the probabilities in cell association.

We denote each individual cell state by $\mathbf{x}_{k}=\left[x_{k}, y_{k}, \dot{x}_{k}, \dot{y}_{k}\right]^{T}$, where $x_{k}$ and $y_{k}$ are the estimated cell centroid positions in the $x$ - and $y$-axes, respectively, and $\dot{x}_{k}$ and $\dot{y}_{k}$ are their corresponding velocities. The system covariance at time $k$ is denoted by $\mathbf{P}_{k}$. The observation state is the cell position in $x$ - and $y$-axes obtained in cell detection process, denoted by $\mathbf{z}_{k}=\left[x_{k}, y_{k}\right]^{T}$.

The backward Kalman filter consists of two steps: backward prediction and update. The prediction equations for the prior state estimate $\hat{\mathbf{x}}_{k-1 \mid k}$ and corresponding system covariance estimate $\mathbf{P}_{k-1 \mid k}$ are

$\hat{\mathbf{x}}_{k-1 \mid k}=\mathbf{F} \hat{\mathbf{x}}_{k \mid k}$,

$\mathbf{P}_{k-1 \mid k}=\mathbf{F P}_{k \mid k} \mathbf{F}^{T}+\mathbf{Q}$,

and the update equations for the posterior cell estimate and covariance estimate are

$\hat{\mathbf{x}}_{k-1 \mid k-1}=\hat{\mathbf{x}}_{k-1 \mid k}+\mathbf{K}_{k-1} \tilde{\mathbf{y}}_{k-1}$

$\mathbf{P}_{k-1 \mid k-1}=\left(\mathbf{I}-\mathbf{K}_{k-1} \mathbf{H}\right) \mathbf{P}_{k-1 \mid k}$

where $\mathbf{F}$ is the state transition model, $\mathbf{Q}$ is the covariance matrix of the process noise, $\mathbf{H}$ is the observation model, which maps the state space into the observation space, $\mathbf{R}$ is the covariance of the observation noise, $\tilde{\mathbf{y}}_{k-1}$ is the innovation or the residual error between the predicted and observed estimate, $\mathbf{S}_{k-1}$ is the innovation covariance, and $\mathbf{K}_{k-1}$ is the optimal Kalman gain matrix, yielding the minimum mean square error. $\tilde{\mathbf{y}}_{k-1}, \mathbf{S}_{k-1}$, and $\mathbf{K}_{k-1}$ are calculated as follows:

$\tilde{\mathbf{y}}_{k-1}=\mathbf{z}_{k-1}-\mathbf{H} \hat{\mathbf{x}}_{k-1 \mid k}$,

$\mathbf{S}_{k-1}=\mathbf{H} \mathbf{P}_{k-1 \mid k} \mathbf{H}^{T}+\mathbf{R}$,

$\mathbf{K}_{k-1}=\mathbf{P}_{k-1 \mid k} \mathbf{H}^{T}\left(\mathbf{H} \mathbf{P}_{k-1 \mid k} \mathbf{H}^{T}+\mathbf{R}\right)^{-1}$.

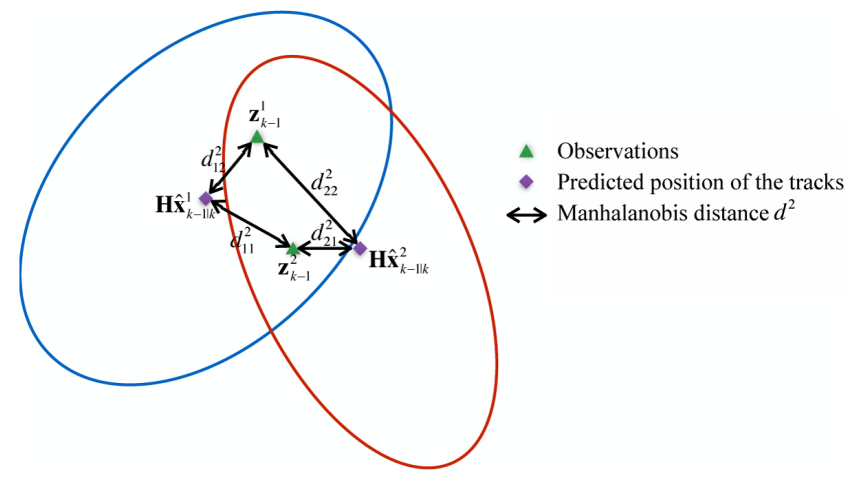

Fig. 7 Backward Kalman filter with validation gating in data association: blue and red ellipses are the validation gate for tracks 1 and 2 , respectively. Track 1 is associated with observation 1 and track 2 is associated with observation 2 in this plot.

We assume a constant velocity model; hence, we have

$\mathbf{F}=\left[\begin{array}{cccc}1 & 0 & \Delta t & 0 \\ 0 & 1 & 0 & \Delta t \\ 0 & 0 & 1 & 0 \\ 0 & 0 & 0 & 1\end{array}\right]$ and $\mathbf{H}=\left[\begin{array}{cccc}1 & 0 & 0 & 0 \\ 0 & 1 & 0 & 0\end{array}\right]$

where $\Delta t=-1$ day.

Backward Kalman filter with validation gating is a popular approach to update the cell state during cell association. In each image, we extract multiple cells, which will be updated to multiple tracks. To associate an observation to a particular track, we employ a validation gate as an association threshold of whether to accept or reject an observation-to-target association. Observations that fall within the validation gate will be updated. As shown in Fig. 7, the blue and red ellipse are the validation gate for cell 1 and cell 2, respectively. Specifically, we compute the Mahalanobis distance between each observation to each state estimate as

$d_{i j}^{2}=\tilde{\mathbf{y}}_{i j, k-1}^{T} \mathbf{S}_{i j, k-1}^{-1} \tilde{\mathbf{y}}_{i j, k-1}$,

where $\tilde{\mathbf{y}}_{i j, k-1}$ is the innovation between the backpredicted track $i$ and the observation $j$ and $\mathbf{S}_{i j, k-1}$ is the corresponding innovation covariance. The observation, whose Mahalanobis distance with a track is smaller than a chi-squared threshold (validation gate), is more likely belonging to this track. If there are no observations within this gate, no observation updates to this track will occur. When there are multiple observations in a validation gate, the estimate is updated with only the best-matched observation (smallest Mahalanobis distance). For instance, observation 1 is associated with track 1 and observation 2 is associated with track 2 in Fig. 7.

The alternative way for multiple observations problem is to apply MHT, where the association of observations can be delayed till sufficient information is available. The flowchart in Fig. 8 shows a diagram of the proposed cell tracking system.

At the end point, which is the initial time point of the backward Kalman filter, we create one track for each cell. Each cell state and covariance estimate at time $k$ are backpropagated to time $k-1$ using Eqs. (2) and (3). If there are $N_{k-1}$ observations which fall within the validation gate, MHT updates all the 


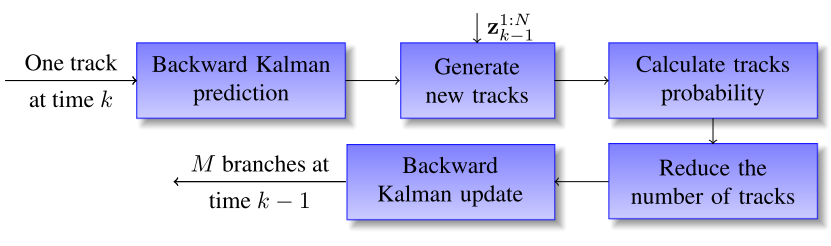

Fig. 8 Flowchart of multiple hypothesis Kalman filtering. Cell state, system covariance, and probabilities of all the tracks are recursively updated over time to form multiple hypotheses. The cell associations are obtained by selecting the most probable hypotheses.

observations to the same track by generating $N_{k-1}$ new branches to the track. Figure 9 provides an example of the MHT process for one cell in two successive time steps. Within the blue ellipse, the purple diamond represents the predicted position of one track at time $k-1$. The green triangles indicate the observations that may be associated with this track with probability $p_{1}, p_{2}$, and $p_{3}$, respectively. The red pentagon depicts the hypothesis (with probability $p_{0}$ ) that the track is coming from the cellgel interface. All the possible hypotheses in step $k-1$ are maintained and considered independently to generate new hypotheses in the next time step, as indicated by the red ellipses in Fig. 9. Since the number of branches for each track increases exponentially, to keep the computation under control, we maintain $M$ branches for each time by including biological knowledge and pruning the tracks with low probability.

Let us consider cell $i$ at time $k$. This cell might be due to the migration or proliferation of an existing cell labeled as $j$ at time $k-1$. It is also possible that this cell is in the cell-gel interface or out of focus (not in the focal plane) at time $k-1$. The corresponding probabilities are calculated in different ways.

We employ the Mahalanobis distance to calculate the probability that track $i$ stems from the migration or proliferation of an existing cell $j$. If the distance is smaller than the threshold gate value, the probability that the track $i$ is associated with observation $j$ is calculated as

$p_{i}\left(\mathbf{z}_{k-1}^{j}\right) \propto\left|2 \pi \mathbf{S}_{i j, k-1}\right|^{-\frac{1}{2}} e^{-\frac{1}{2} d_{i j}^{2}}$

We assume that the cells nearer to the cell-gel interface (larger $y$-position) are more likely to be new tracks. The probability that track $i$ is due to migration from the cell-gel interface is computed as

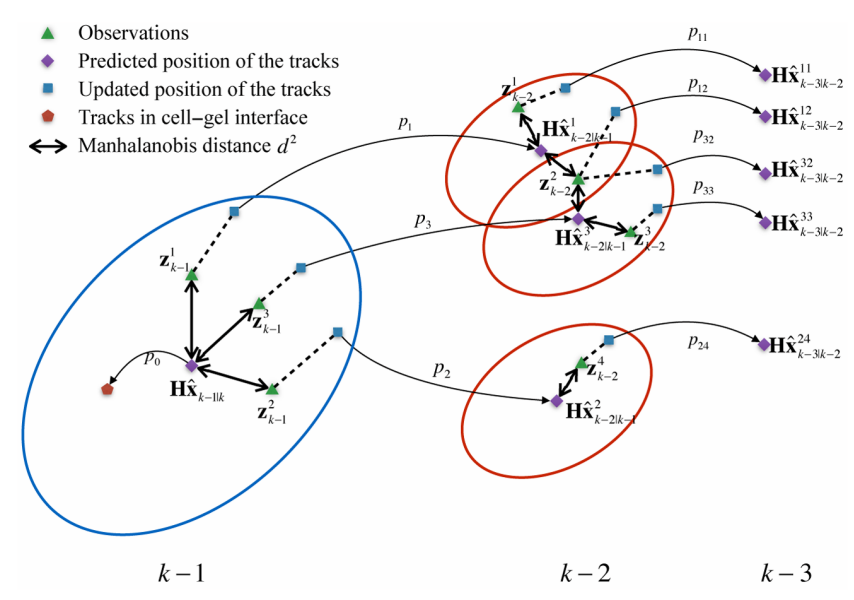

Fig. 9 MHT for one cell in two successive time steps.
$p_{i}(\mathrm{M}) \propto \frac{1}{\left(b_{1}-y_{k-1 \mid k}\right)^{b_{2}}}$,

where $b_{1}=1700$ and $b_{2}=2.47$ are the empirical parameters tuned from our experimental data and $y_{k-1 \mid k}$ is the cell position in $y$-direction from backward Kalman prediction. The probabilities for track $i$ estimated by Eqs. (11) and (12) are then normalized for this time step.

We include biological knowledge to reduce the number of the new tracks in each step. Since the doubling time of our ECs is 15 to $48 \mathrm{~h}$, we assume that one cell can at most split once within one day. In other words, we allow at most two cells at time $k$ to be associated with one same observation at time $k-1$ during merging. In our angiogenic vessels, cells cannot overtake each other due to the existence of tight cell junctions, so we eliminate the tracks where overtaking happens during association. Meanwhile, we discard the tracks with low probability to keep the number of tracks generation computations under control.

As mentioned, we keep $M$ branches and obtain their corresponding cell state estimates and system covariance estimates with backward Kalman update for each time step. Typically, $M=4$ is selected since the remaining branches have negligible probabilities. Each of these new tracks is considered independently and used to generate new predictions for next time step. We repeat this tracking process until $k=1$ and obtain $N_{0} M^{t-1}$ hypotheses in total, where $N_{0}$ is the number of the cells obtained from the end-point confocal images and $t$ is the total time steps. Last, the tracks are selected as the most probable hypotheses.

\section{Results and Discussion}

We applied the proposed automated multicell tracking system to the experimental time-lapse phase-contrast images with the aim of tracking the nuclei of the ECs within the angiogenic vessels.

\subsection{Image Registration Results}

With our proposed registration algorithm, we aligned all the phase-contrast image sequences and their corresponding endpoint confocal images. We overlay the end-point phase-contrast images and their corresponding end-point confocal images to illustrate the registration results (see Fig. 10).

Figures 10(a) and 10(d) are the original end-point experimental images obtained from phase-contrast microscopy. Figures 10(b) and 10(e) are their corresponding confocal images. Here, the blue blobs are the cell nuclei stained by Hoechst, and the green structures are the actin stained by Phalloidin. We can see that the size and position of the vessels are different in the phase-contrast images and confocal images. The aligned phase-contrast and confocal images are shown in Figs. 10(c) and 10(f), where the resized phase-contrast images are shown in the background. We marked the cell nuclei stained in the confocal images as green blobs for clearer identification. The angiogenic structures are well-aligned in Fig. 10, suggesting our image registration algorithm is accurate.

\subsection{Cell Detection Results}

We consider the same examples as in Sec. 3.1 to illustrate our cell detection results. Figures 11(a) and 11(e) show the results for the proposed CNN-based cell detection algorithm. Figures 11(b) and 11(f) show the results from partial least square regression (PLSR) approach with 30 PLS components as explained in Ref. 47. Figures $11(\mathrm{c})$ and $11(\mathrm{~g})$ show the cell 


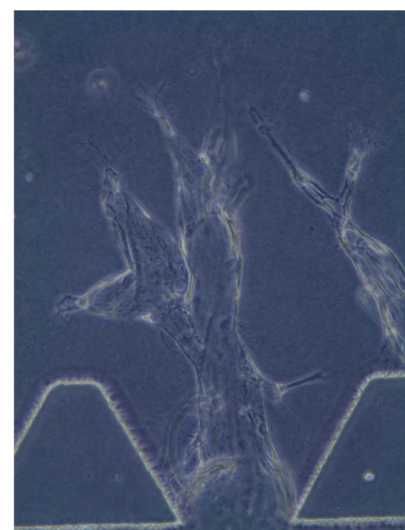

(a)

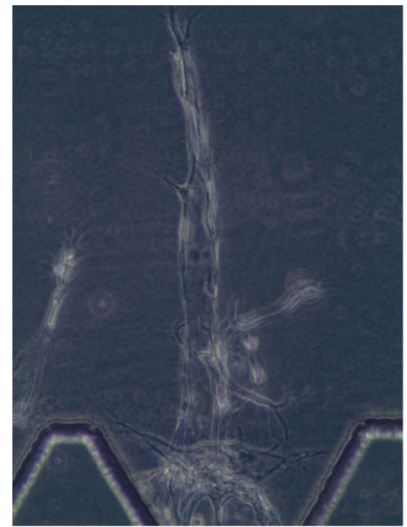

(d)

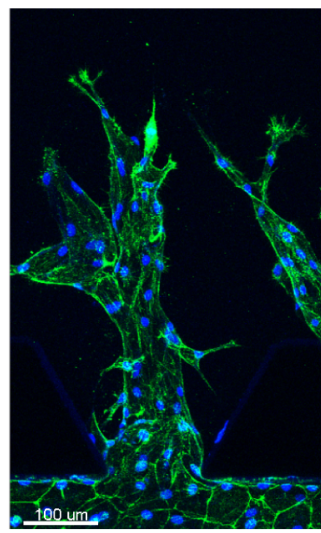

(b)

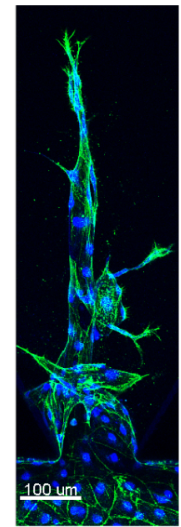

(e)

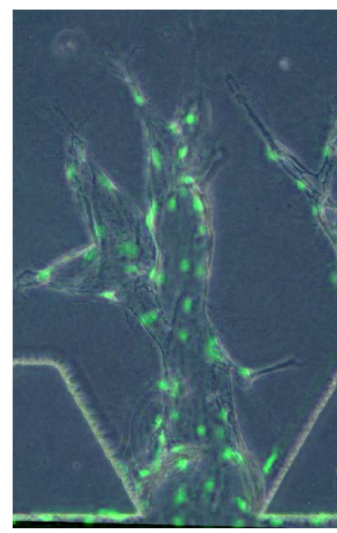

(c)

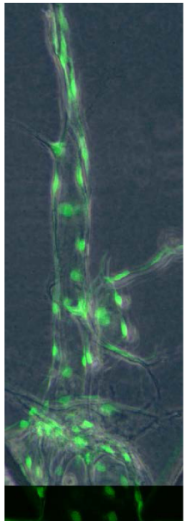

(f)

Fig. 10 Illustration of image registration results: (a) and (d) are the end-point phase-contrast images, (b) and (e) are their corresponding confocal images, and (c) and (f) show the coregistered confocal and phase-contrast images.

detection results for principal component analysis (PCA) in combination with SVM classification. We also trained an SVM classifier based on the original high-dimensional template data to distinguish the cells from the background in our experimental images, for comparison. The results are shown in Figs. 11(d) and 11(h). The red stars $(*)$ label the location of the detected cell nuclei for all these images.

To evaluate the performance of these approaches quantitatively, precision and recall measures are calculated. Precision is the fraction of detected cells that are actual cells, and recall is the fraction of the actual cells that are detected

Precision $=\frac{\mathrm{TP}}{\mathrm{TP}+\mathrm{FP}} \quad$ and $\quad$ Recall $=\frac{\mathrm{TP}}{\mathrm{TP}+\mathrm{FN}}$,

where TP, FP, and FN are defined in the $2 \times 2$ confusion matrix in Table $1 .^{48}$ This confusion matrix describes the four possible outcomes of a given binary classifier and a set of instances.

Table 1 Confusion matrix for a binary classifier. ${ }^{48}$

\begin{tabular}{lcc} 
& Actual positive & Actual negative \\
\hline Assigned positive & True positive (TP) & False positive (FP) \\
Assigned negative & False negative (FN) & True negative (TN) \\
\hline
\end{tabular}

To assess the different cell detection approaches, we also compute $F$-score $F_{1}$, defined as the harmonic mean of precision and recall

$F_{1}=2 \cdot \frac{\text { Precision } \cdot \text { Recall }}{\text { Precision }+ \text { Recall }}$

To validate the cell detection results, we use the end-point phase-contrast images, matching the estimated cell locations with their corresponding confocal images. We analyzed 18 endpoint phase-contrast images in this paper. Since the phasecontrast image is a 2-D slice and the confocal image is in 3-D with multiple slices, we choose the ground truth in two ways. For the first case, the actual positives and negatives are extracted from all the slices of confocal images. However, since our phase-contrast image is in 2-D, we use only one slice of the confocal image to provide the ground truth for the second case. We choose the slice that has a similar focal plane as the 2-D phase-contrast image. The TP, FP, and FN values for the four approaches are provided in Table 2 . Their corresponding precision, recall, and $F_{1}$ values are listed in Table 3 .

For case 1 , all the precision values are above $80 \%$, suggesting that most of the detected cells are actual cells. The recall for CNN reaches $85.7 \%$, which is higher than for the other three approaches, indicating that $\mathrm{CNN}$ detects most of the actual cells. 


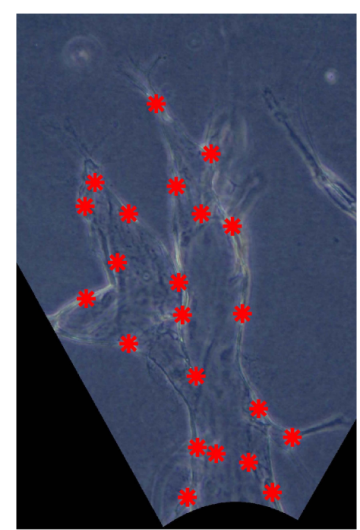

(a)

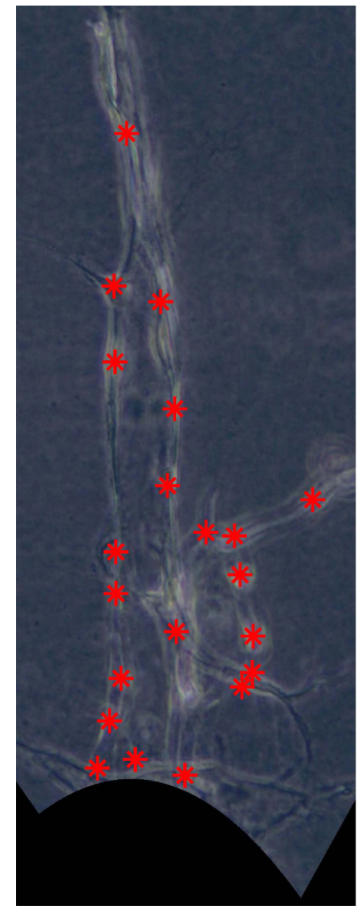

(e)

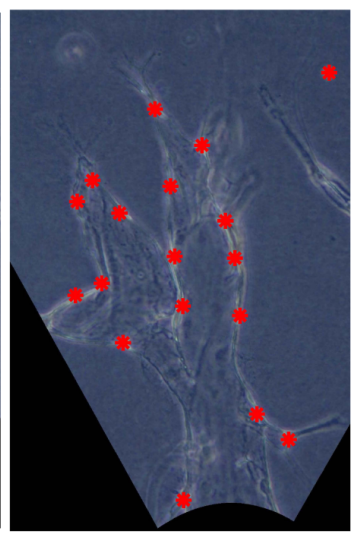

(b)

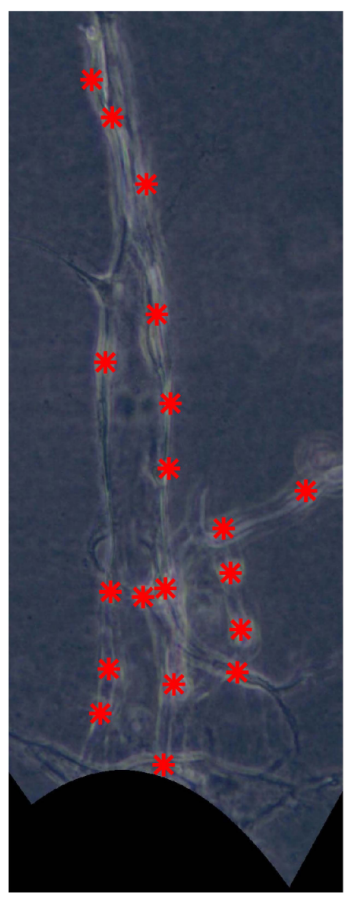

(f)

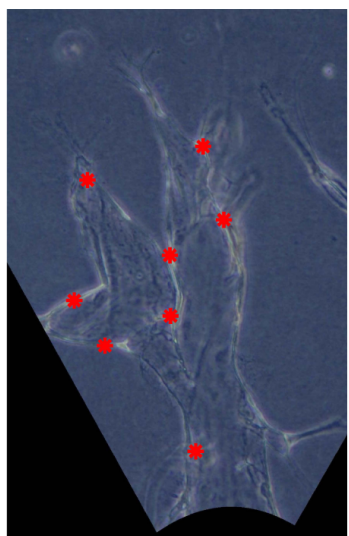

(c)

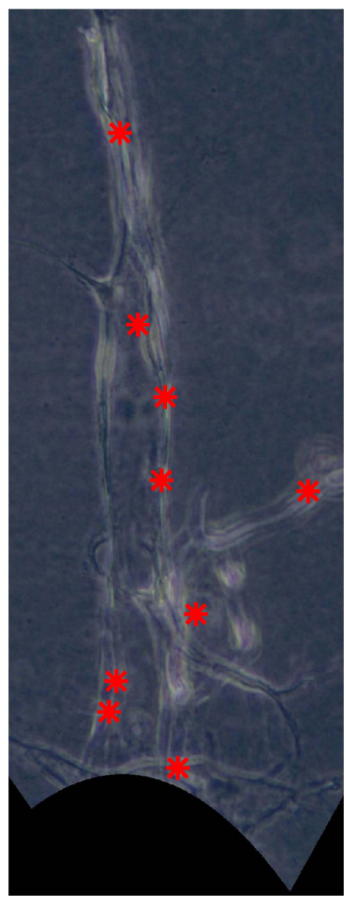

(g)

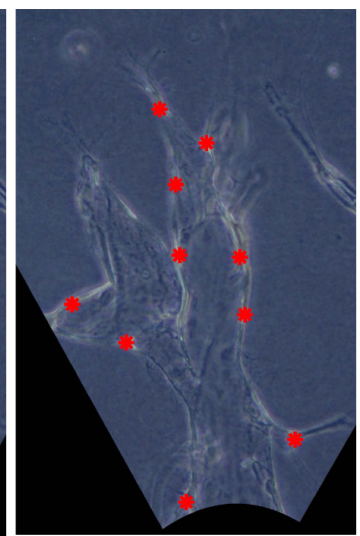

(d)

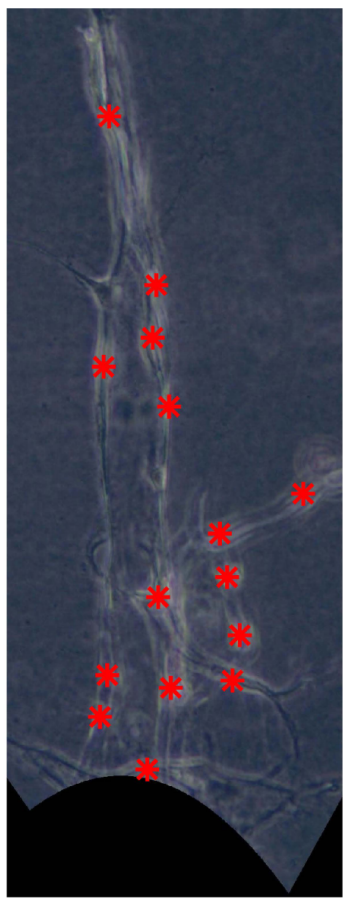

(h)

Fig. 11 Cell detection results of examples in Fig. 10 from different approaches: (a) and (e) from CNN classifier, (b) and (f) from PLSR, ${ }^{47}$ (c) and (g) from PCA in conjunction with SVM, and (d) and (h) from an SVM classifier. Red stars $(*)$ indicate the locations of the detected cell nuclei.

Table 2 TP, FP, and FN values for different cell detection approaches.

\begin{tabular}{|c|c|c|c|c|c|c|}
\hline \multirow[b]{2}{*}{ Detection approaches } & \multicolumn{3}{|c|}{ Case 1: all slices } & \multicolumn{3}{|c|}{ Case 2: one slice } \\
\hline & TP & FP & FN & TP & FP & $\mathrm{FN}$ \\
\hline CNN & 228 & 33 & 38 & 228 & 33 & 22 \\
\hline PLSR & 217 & 38 & 49 & 217 & 38 & 33 \\
\hline PCA and SVM & 107 & 26 & 159 & 107 & 26 & 143 \\
\hline SVM & 124 & 23 & 142 & 124 & 23 & 126 \\
\hline
\end{tabular}

Table 3 Classification performance for different cell detection approaches.

\begin{tabular}{lcccccccc}
\hline & \multicolumn{3}{c}{ Case 1: all slices } & & \multicolumn{3}{c}{ Case 2: one slice } \\
\cline { 2 - 4 } \cline { 7 - 8 } Approaches & $\begin{array}{c}\text { Precision } \\
(\%)\end{array}$ & $\begin{array}{c}\text { Recall } \\
(\%)\end{array}$ & $F_{1}$ & & $\begin{array}{c}\text { Precision } \\
(\%)\end{array}$ & $\begin{array}{c}\text { Recall } \\
(\%)\end{array}$ & $F_{1}$ \\
\hline CNN & 87.4 & 85.7 & 0.87 & 87.4 & 91.2 & 0.89 \\
PLSR & 85.1 & 81.6 & 0.83 & 85.1 & 86.8 & 0.86 \\
PCA and & 80.5 & 40.2 & 0.54 & 80.5 & 42.8 & 0.56 \\
SVM & & & & & & \\
SVM & 84.4 & 46.6 & 0.60 & 84.4 & 49.6 & 0.62 \\
\hline
\end{tabular}


The recall of $\mathrm{CNN}$ is limited to about $85.7 \%$, which can be explained as follows. The height of our device is around $120 \mu \mathrm{m}$ and there are cells migrating throughout this range. We only acquire the 2-D image of the angiogenic vessels in focus. Some cells, which are external to the vessels, are out of focus and almost invisible in the phase-contrast images. These cells are not detected by our algorithms. However, these out-of-focus cells are observed in the 3-D confocal images. This scenario can be seen in Fig. 10.

For case 2, the recall for all the four approaches increases as shown in Table 3. Particularly, the recall for the proposed cell detection algorithm (CNN) increases to $91.2 \%$. From the values of $F$-score in Table 3, which balances recall and precision, we can also conclude that $\mathrm{CNN}$ is a preferable algorithm to detect the ECs within the 3-D angiogenic vessels from our experimental phase-contrast images. We hypothesize that the features extracted from $\mathrm{CNN}$ are more suitable to represent the cell templates.

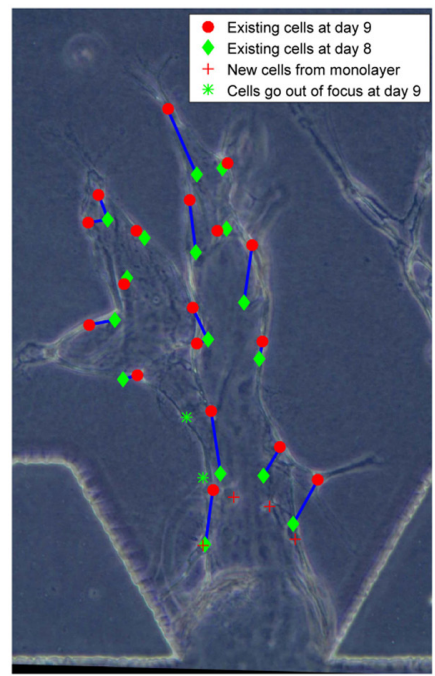

(a)

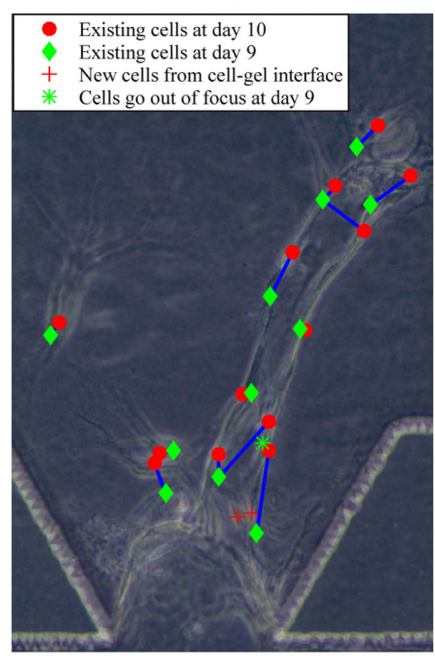

(e)

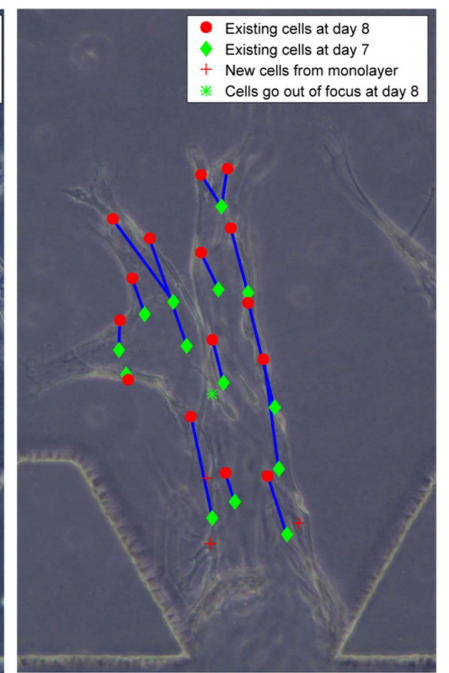

(b)

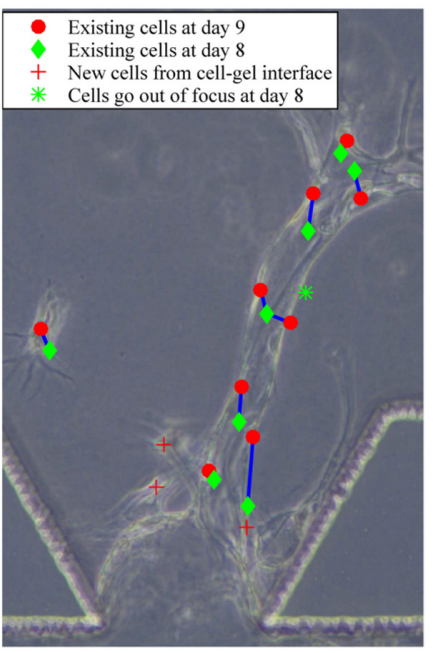

(f)

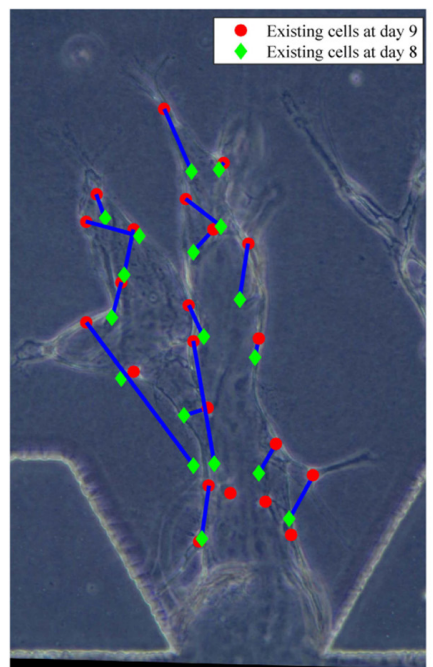

(c)

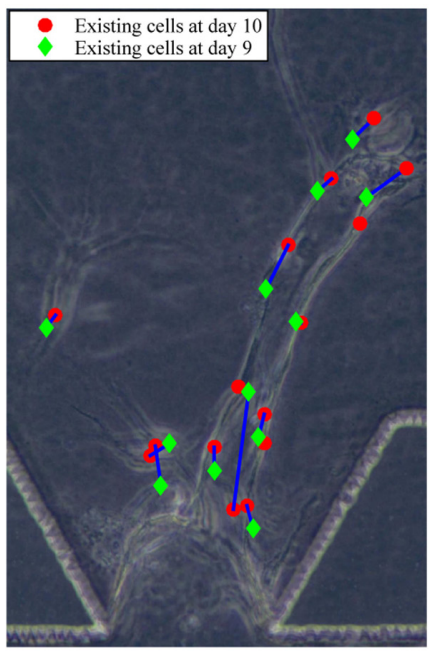

(g)

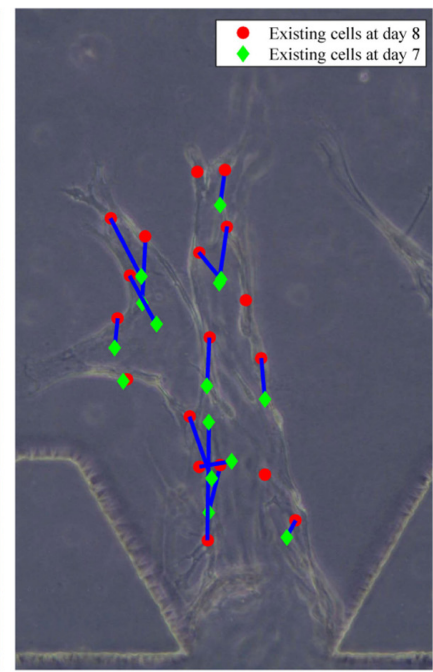

(d)

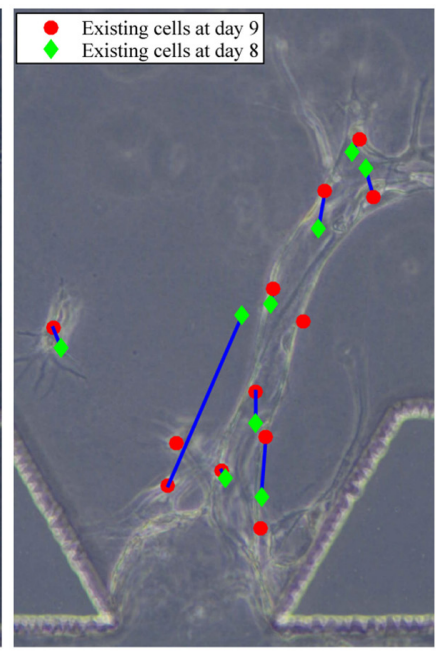

(h)

Fig. 12 Cell tracking examples: (a)-(d) for example 1 and (e)-(h) for example 2. (a), (b) and (e), (f): backward Kalman filter with MHT, (c), (d) and (g), (h): backward Kalman filter with validation gating. Each image shows the outcomes of the tracking algorithms for two successive frames, where the phase-contrast image is shown in the background. 
Table 4 Accuracy (percentage of correct associations) for different cell association approaches. I, nearest-neighborhood association; II, Kalman filter with validation gating; III, Hungarian method; and IV, Kalman filter with MHT.

\begin{tabular}{cccc} 
& Correct associations & Total associations & $\begin{array}{c}\text { Association } \\
\text { accuracy (\%) }\end{array}$ \\
\hline I & 53 & 198 & 26.7 \\
II & 119 & 198 & 60.1 \\
III & 105 & 198 & 53.0 \\
IV & 171 & 198 & 86.4 \\
\hline
\end{tabular}

the proposed approach, the nearest-neighborhood association, the backward Kalman filter with validation gating, and the Hungarian method ${ }^{32}$ (see Table 4). We excluded in this comparison methods in the literature that require more information about the cells than merely the cell locations, since such information is unavailable for our data. Since these methods cannot be applied to our data, we did not assess them. We obtained the ground truth through manual association, which is the traditional method of analyzing the cell migratory behaviors.

Since the sampling interval is one day, cells migrate over a relatively long distance between frames. Using nearest-neighborhood association, the new tracks at time $k$ are typically associated wrongly to cells that are near to the cell-gel interface at time $k-1$. In the backward Kalman filter with validation gating approach, we predict the track backward each time step via a constant velocity motion model. In this model, we assume that the track velocity is a constant during one time step, and we update it at every time step. This approach yields more reliable association results, improving the association accuracy from $26.7 \%$ to $60.1 \%$. In the Hungarian method, we first predict the new positions of the cells at time $k$ using a constant velocity model and then estimate the Euclidean distances of the predicted cells at time $k$ and the cells at time $k-1$, to find the associations with minimal cost. However, cell migration and proliferation are not considered since it only allows for one-to-one matching. Thus, only $53.0 \%$ association accuracy is obtained. In our approach, we combine MHT with backward Kalman filter, which delays the decision making, and determine the associations once sufficient information is available. Moreover, biological knowledge is incorporated when considering the new cells from the cell-gel interface and reducing the number of new tracks. As a result, we can track not only the migration and proliferation of the existing cells but also the new cells from cell-gel interface and out-of-focus plane (see Fig. 12). This explains why the association accuracy increases to $86.4 \%$ for our approach. Compared with the association accuracy $(85.9 \%)$ based on PLSR in Ref. 47, we can also conclude that the increment in cell detection accuracy due to the CNN improves the association accuracy.

Cell trajectory information can be obtained from the tracking results. Moreover, the system can automatically generate cell lineage plots (see Fig. 13), showing the history of the cell proliferation and cell migration into the gel, with timestamps of when it is in focus and out of focus. Such cell lineage plot provides crucial information (e.g., the number of cells, division time, growth fraction, etc.) to biologists who are interested in studying cell migration behaviors under different conditions.

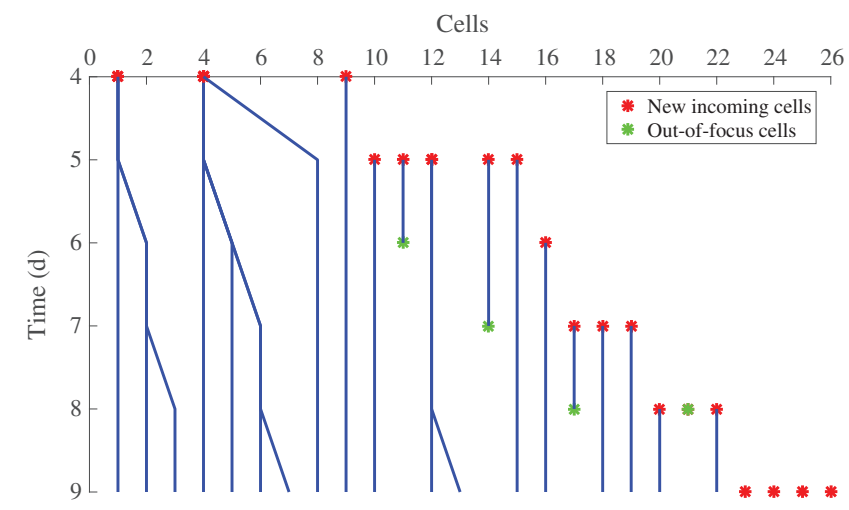

(a)

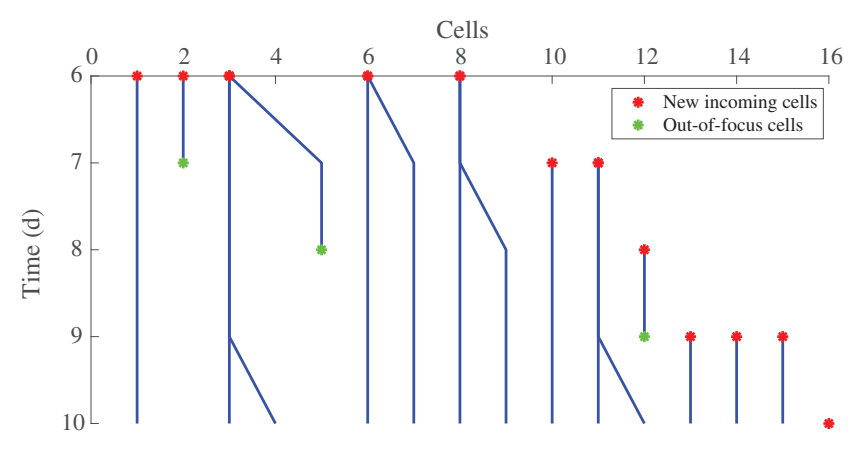

(b)

Fig. 13 Cell lineage plot: (a) for example 1 and (b) for example 2 in Fig. 12. The blue lines represent the cells that migrate within the 3-D gel; the red stars represent the incoming cells from the cell-gel interface, whereas the green stars indicate the cells migrating out of focus.

The cell lineage plots in Figs. 13(a) and 13(b) correspond to the two examples in Fig. 12. The blue lines indicate that cells migrate within the 3-D gel. The branch points of these blue lines represent cell proliferation. The red stars illustrate the incoming cells from the cell-gel interface. The green stars indicate that cells migrate out of focus. The cell lineage plot illustrates the history of cell migration and proliferation in a compact and effective manner.

By comparing the cell lineage plots obtained under different experimental conditions, biologists can easily figure out the influences of the chemical factors on cell division and cell migration. For instance, Fig. 13(a) is from angiogenic experiment under positive sphingosine-1-phosphate (S1P) gradient, and Fig. 13(b) is from angiogenic experiment without S1P. ${ }^{9}$ By comparing these two figures, one can conclude that positive S1P gradient induces more recruitment of cells from the monolayer and stimulates cell proliferation. The cell lineage plots provide quantitative information about the influence of S1P on angiogenesis. Such quantitative data are an important step toward better understanding and new theories of angiogenesis; hence, the proposed tools probably will enable biologists to make new discoveries concerning angiogenesis. These data can also be used to develop mathematical models to predict the cell migration under different conditions, leading ultimately to a better understanding cell migration in angiogenesis.

\section{Conclusion}

We presented an automated image analysis system to track the migrating ECs within 3-D angiogenic vessels cultured in MFDs by combining end-point confocal and time-lapse phase-contrast 
images. This system consists of image registration, vessel segmentation, cell detection, and multiple hypothesis Kalman filtering to track cell proliferation and migration (in/out of focus) in 3-D angiogenic vessels. We incorporate biological knowledge, such as vessel information and experimental device properties, to add constraints and to estimate the track probability during cell association. Our proposed cell detection algorithm based on CNN yields $87.4 \%$ precision and $91.2 \%$ recall for cell candidates detection, which helps the biologists to recognize a large number of cells automatically with high accuracy. The association accuracy reaches $86.4 \%$ for the proposed multiple hypothesis Kalman filtering approach when associating the detected cell candidates. In the future, we will apply deep residual learning to improve cell detection accuracy. We will also explore other possible features of cells and combine the detection likelihood of each cell with the Mahalanobis distance to improve the association accuracy. From the tracking results (see Fig. 12), we are able to obtain information about the cell trajectory and create a cell lineage plot (see Fig. 13) to visualize the history of the cell migration and proliferation in a compact and effective manner. By comparing with the identified biomarkers in the end-point confocal images, biologists can explore links between the chemical and physical characteristics of a cell. With this information about the cell history, mathematical models can be developed to predict the cell migration under different cell conditions, leading ultimately to a better understanding of the biological processes in cells.

\section{Disclosures}

No conflicts of interest, financial or otherwise, are declared by the authors.

\section{Acknowledgments}

This research was supported by the National Research Foundation Singapore through the Singapore MIT Alliance for Research and Technology's BioSystems and Micromechanics Interdisciplinary Research program.

\section{References}

1. W. A. Farahat et al., "Ensemble analysis of angiogenic growth in threedimensional microfluidic cell cultures," PLoS One 7(5), e37333 (2012).

2. H. Gerhardt, "VEGF and endothelial guidance in angiogenic sprouting," in VEGF in Development, H. Gerhardt, Ed., pp. 68-78, Springer, New York (2008).

3. A. Das et al., "A hybrid continuum-discrete modelling approach to predict and control angiogenesis: analysis of combinatorial growth factor and matrix effects on vessel-sprouting morphology," Philos. Trans. R. Soc. Ser. A 368(1921), 2937-2960 (2010).

4. F. De Smet et al., "Mechanisms of vessel branching filopodia on endothelial tip cells lead the way," Arterioscler. Thromb. Vasc. Biol. 29(5), 639-649 (2009).

5. J. Folkman and C. Haudenschild, "Angiogenesis in vitro," Nature 288, 551-556 (1980).

6. L.-L. S. Ong et al., "A Bayesian filtering approach to incorporate 2D/3D time-lapse confocal images for tracking angiogenic sprouting cells interacting with the gel matrix," Med. Image Anal. 18(1), 211-227 (2014).

7. F. Progatzky, M. J. Dallman, and C. L. Celso, "From seeing to believing: labelling strategies for in vivo cell-tracking experiments," Interface Focus 3(3), 20130001 (2013).

8. D. B. Murphy, Fundamentals of Light Microscopy and Electronic Imaging, John Wiley and Sons, New York (2002).

9. M. Wang et al., "Automated tracking and quantification of angiogenic vessel formation in 3D microfluidic devices," PLoS One 12(11), e0186465 (2017).
10. A. Dufour et al., "Segmenting and tracking fluorescent cells in dynamic 3-D microscopy with coupled active surfaces," IEEE Trans. Image Process. 14(9), 1396-1410 (2005).

11. D. Padfield et al., "Spatio-temporal cell cycle phase analysis using level sets and fast marching methods," Med. Image Anal. 13(1), 143-155 (2009).

12. A. Sacan, H. Ferhatosmanoglu, and H. Coskun, "Celltrack: an opensource software for cell tracking and motility analysis," Bioinformatics 24(14), 1647-1649 (2008).

13. F. Amat et al., "Fast, accurate reconstruction of cell lineages from largescale fluorescence microscopy data," Nat. Methods 11, 951-958 (2014).

14. M. Schiegg et al., "Graphical model for joint segmentation and tracking of multiple dividing cells," Bioinformatics 31(6), 948-956 (2015).

15. I. Ersoy et al., "Cell segmentation using Hessian-based detection and contour evolution with directional derivatives," in 15th IEEE Int. Conf. on Image Processing, pp. 1804-1807, IEEE (2008).

16. F. Ambriz-Colin et al., "Detection of biological cells in phase-contrast microscopy images," in Fifth Mexican Int. Conf. on Artificial Intelligence, pp. 68-77, IEEE (2006).

17. C.-H. Huang et al., "Online 3-D tracking of suspension living cells imaged with phase-contrast microscopy," IEEE Trans. Biomed. Eng. 59(7), 1924-1933 (2012).

18. T. Kanade et al., "Cell image analysis: algorithms, system and applications," in IEEE Workshop on Applications of Computer Vision (WACV), pp. 374-381, IEEE (2011).

19. H. Su et al., "Phase contrast image restoration via dictionary representation of diffraction patterns," Lect. Notes Comput. Sci. 7512, 615-622 (2012).

20. Z. Yin, T. Kanade, and M. Chen, "Understanding the phase contrast optics to restore artifact-free microscopy images for segmentation," Med. Image Anal. 16, 1047-1062 (2012).

21. D. Young et al., "Towards automatic cell identification in DIC microscopy," J. Microsc. 192(2), 186-193 (1998).

22. R. C. Gonzalez, Digital Image Processing, Pearson Education, New Delhi, India (2009).

23. N. Wei et al., "An in situ probe for on-line monitoring of cell density and viability on the basis of dark field microscopy in conjunction with image processing and supervised machine learning," Biotechnol. Bioeng. 97(6), 1489-1500 (2007).

24. J. W. Han et al., "The application of support vector machine classification to detect cell nuclei for automated microscopy," Mach. Vision Appl. 23(1), 15-24 (2012).

25. M. Wang et al., "Novel cell segmentation and online SVM for cell cycle phase identification in automated microscopy," Bioinformatics 24(1), 94-101 (2008).

26. Z. Yin et al., "Cell segmentation in microscopy imagery using a bag of local Bayesian classifiers," in IEEE Int. Symp. on Biomedical Imaging: From Nano to Macro, pp. 125-128, IEEE (2010).

27. X. Chen, X. Zhou, and S. T. Wong, "Automated segmentation, classification, and tracking of cancer cell nuclei in time-lapse microscopy," IEEE Trans. Biomed. Eng. 53(4), 762-766 (2006).

28. Z. N. Demou and L. V. McIntire, "Fully automated three-dimensional tracking of cancer cells in collagen gels determination of motility phenotypes at the cellular level," Cancer Res. 62(18), 5301-5307 (2002).

29. I. Adanja et al., "A new method to address unmet needs for extracting individual cell migration features from a large number of cells embedded in 3D volumes," PLoS One 6(7), e22263 (2011).

30. I. Adanja et al., "Automated tracking of unmarked cells migrating in three-dimensional matrices applied to anti-cancer drug screening," Exp. Cell. Res. 316(2), 181-193 (2010).

31. O. Debeir et al., "Tracking of migrating cells under phase-contrast video microscopy with combined mean-shift processes," IEEE Trans. Med. Imaging 24(6), 697-711 (2005).

32. H. Kuhn, "The Hungarian method for the assignment problem," Nav. Res. Logist. 52(1), 7-21 (2005).

33. K. Magnusson and J. Jalden, "A batch algorithm using iterative application of the Viterbi algorithm to track cells and construct cell lineages," in 9th IEEE Int. Symp. on Biomedical Imaging (ISBI) (2012).

34. X. Yang, H. Li, and X. Zhou, "Nuclei segmentation using markercontrolled watershed, tracking using mean-shift, and Kalman filter in time-lapse microscopy," IEEE Trans. Circuits Syst. Regul. Pap. 53(11), 2405-2414 (2006). 
35. I. Smal, W. Niessen, and E. Meijering, "Particle filtering for multiple object tracking in molecular cell biology," in IEEE Nonlinear Statistical Signal Processing Workshop, pp. 129-132, IEEE (2006).

36. F. Bunyak et al., "Quantitative cell motility for in vitro wound healing using level set-based active contour tracking," in 3rd IEEE Int. Symp. on Biomedical Imaging: Nano to Macro, pp. 1040-1043, IEEE (2006).

37. L.-L. S. Ong, M. H. Ang, and H. H. Asada, "Tracking of cell population from time lapse and end point confocal microscopy images with multiple hypothesis Kalman smoothing filters," in IEEE Computer Society Conf. on Computer Vision and Pattern Recognition Workshops (CVPRW), pp. 71-78, IEEE (2010).

38. X. Lou, M. Schiegg, and F. Hamprecht, "Active structured learning for cell tracking: algorithm, framework, and usability," IEEE Trans. Med. Imaging 33, 849-860 (2014).

39. E. C. Sherbrooke, N. M. Patrikalakis, and E. Brisson, "An algorithm for the medial axis transform of 3D polyhedral solids," IEEE Trans. Visual Comput. Graphics 2(1), 44-61 (1996).

40. N. Otsu, "A threshold selection method from gray-level histograms," IEEE Trans. Syst. Man Cybern. 9(1), 62-66 (1979).

41. S. Hijazi, R. Kumar, and C. Rowen, Using Convolutional Neural Networks for Image Recognition, Cadence Design Systems Inc., San Jose, California (2015).

42. A. Vedaldi and K. Lenc, "Matconvnet: convolutional neural networks for MATLAB," in Proc. of the 23rd ACM Int. Conf. on Multimedia, pp. 689-692, ACM (2015).

43. V. Nair and G. E. Hinton, "Rectified linear units improve restricted Boltzmann machines," in Proc. of the 27th Int. Conf. on Machine Learning (ICML), pp. 807-814 (2010).

44. A. Krizhevsky, I. Sutskever, and G. E. Hinton, "ImageNet classification with deep convolutional neural networks," in Advances in Neural Information Processing Systems, pp. 1097-1105 (2012).

45. N. Srivastava et al., "Dropout: a simple way to prevent neural networks from overfitting," J. Mach. Learn. Res. 15(1), 1929-1958 (2014).

46. M. B. Christopher, Pattern Recognition and Machine Learning, Springer-Verlag, New York (2016).
47. M. Wang et al., "Automated tracking of cells from phase contrast images by multiple hypothesis Kalman filters," in IEEE Int. Conf. on Acoustics, Speech and Signal Processing (ICASSP), pp. 942-946, IEEE (2015).

48. T. Fawcett, "An introduction to ROC analysis," Pattern Recognit. Lett. 27(8), 861-874 (2006).

Mengmeng Wang received her BEng degree from the School of Chemical and Biomolecular Engineering, Nanyang Technological University (NTU), Singapore, in 2011. She received her PhD from the School of Electrical and Electronic Engineering (EEE) from NTU in 2017, working on quantitative analysis of angiogenic vascular. Currently, she is a research fellow at Energy Research Institute, NTU. Her research interests include machine learning, deep learning, data analysis, and image processing.

Lee-Ling Sharon Ong is a research scientist at Singapore-MIT Alliance for Research and Technology Centre. She received her doctoral degree in mechatronics engineering from the University of Sydney, Australia, in 2008. Her work focuses on integrating biological models with experimental data from time-lapse microscopy. Her research interests include image processing, stochastic modeling, and multiagent systems.

Justin Dauwels is an associate professor at the School of EEE, NTU. $\mathrm{He}$ is also the deputy director of ST Engineering-NTU Corporate Lab and the director of the neuroengineering program at the School of EEE. His research interests are in Bayesian statistics, iterative signal processing, machine learning, and computational neuroscience.

H. Harry Asada is a Ford professor of engineering in the Department of Mechanical Engineering, Massachusetts Institute of Technology, USA. He is also the director of d'Arbeloff Laboratory for Information Systems and Technology and current head of control, instrumentation, and robotics in the Department of Mechanical Engineering, MIT. His expertise ranges from robotics, biomedical engineering, dynamic systems and control, information technology to design and manufacturing. 